OPEN ACCESS

Edited by:

Andrés Navarrete,

National Autonomous University of

Mexico, Mexico

Reviewed by:

Noam Butterfield,

University of British Columbia, Canada

Hady Keita,

University of the South Sierra, Mexico

*Correspondence:

Xiaojuan $\mathrm{Li}$

lixiaojuan@jnu.edu.cn

Jiaxu Chen

chenjiaxu@hotmail.com

${ }^{\dagger}$ These authors share first authorship

Specialty section: This article was submitted to

Ethnopharmacology,

a section of the journal

Frontiers in Pharmacology

Received: 19 October 2020 Accepted: 24 March 2021

Published: 16 April 2021

Citation:

Hao W, Wu J, Yuan N, Gong L, Huang J, Ma Q, Zhu H, Gan H, DaX

Deng L, LiX and Chen J (2021)

Xiaoyaosan Improves AntibioticInduced Depressive-Like and Anxiety-

Like Behavior in Mice Through

Modulating the Gut Microbiota and Regulating the NLRP3 Inflammasome in the Colon.

Front. Pharmacol. 12:619103. doi: $10.3389 /$ fphar.2021.619103

\section{Xiaoyaosan Improves}

Antibiotic-Induced Depressive-Like and Anxiety-Like Behavior in Mice Through Modulating the Gut Microbiota and Regulating the NLRP3 Inflammasome in the Colon

\author{
Wenzhi Hao ${ }^{1+}$, Jiajia Wu ${ }^{2+}$, Naijun Yuan ${ }^{1}$, Lian Gong ${ }^{1}$, Junqing Huang ${ }^{1}$, Qingyu Ma ${ }^{1}$, \\ Huizheng Zhu ${ }^{1}$, Hua Gan ${ }^{1}$, Xiaoli Da ${ }^{1}$, Lijuan Deng ${ }^{1}$, Xiaojuan $\mathrm{Li}^{1 *}$ and Jiaxu Chen ${ }^{1,3 *}$ \\ ${ }^{1}$ Guangzhou Key Laboratory of Formula-Pattern of Traditional Chinese Medicine, Formula-Pattern Research Center, School of \\ Traditional Chinese Medicine, Jinan University, Guangzhou, China, ${ }^{2}$ School of Basic Medical Science, Hubei University of Chinese \\ Medicine, Wuhan, China, ${ }^{3}$ School of Traditional Chinese Medicine, Beijing University of Chinese Medicine, Beijing, China
}

Disturbance of the gut microbiota plays an essential role in mental disorders such as depression and anxiety. Xiaoyaosan, a traditional Chinese medicine formula, has a wide therapeutic spectrum and is used especially in the management of depression and anxiety. In this study, we used an antibiotic-induced microbiome-depleted (AIMD) mouse model to determine the possible relationship between imbalance of the intestinal flora and behavioral abnormalities in rodents. We explored the regulatory effect of Xiaoyaosan on the intestinal flora and attempted to elucidate the potential mechanism of behavioral improvement. We screened NLRP3, ASC, and CASPASE-1 as target genes based on the changes in gut microbiota and explored the effect of Xiaoyaosan on the colonic NLRP3 pathway. After Xiaoyaosan intervention, AIMD mice showed a change in body weight and an improvement in depressive and anxious behaviors. Moreover, the gut flora diversity was significantly improved. Xiaoyaosan increased the abundance of Lachnospiraceae in AIMD mice and decreased that of Bacteroidaceae, the main lipopolysaccharide (LPS)producing bacteria, resulting in decreased levels of LPS in feces, blood, and colon tissue. Moreover, serum levels of the inflammatory factor, IL-1 $\beta$, and the levels of NLRP3, ASC, and CASPASE-1 mRNA and DNA in the colon were significantly reduced. Therefore, Xiaoyaosan may alleviate anxiety and depression by modulating the gut microbiota, correcting excessive LPS release, and inhibiting the immoderate activation of the NLRP3 inflammasome in the colon.

Keywords: Xiaoyaosan, antidepressant, anxiolytic, lipopolysaccharide, gut microbiota 


\section{INTRODUCTION}

Depression and anxiety are the most common psychiatric disorders, affecting more than 350 million people globally (Baxter et al., 2014). These mental disorders can cause significant impairment, increase annual care costs, and represent a significant economic burden worldwide (Kessler and Bromet, 2013). Current drug treatments for these diseases are limited and cause many toxic side effects (Rieder et al., 2017). Extensive evidence indicates that intestinal flora plays an essential role in the pathophysiology of depression and anxiety (Sherwin et al., 2018). Changes in the gut microbiota have been reported in patients with depressive disorder and anxiety (Chen et al., 2018), and similar results have been observed in rodent models with these conditions (Hyo-Min et al., 2018). Probiotic treatment can improve the clinical symptoms of patients with depression and anxiety (Slykerman et al., 2017). These findings indicate that gut microbes are involved in the development of depression and anxiety and could be a potential target for drug development in the management of such conditions. However, the exact role of the intestinal flora in the pathogenesis of depression and anxiety is unknown and the downstream physiological mechanisms by which gut microbes influence human behavior remain unclear (Sender et al., 2016). Recent findings provide new insights into the pathogenesis of depression and anxiety mediated by intestinal flora, suggesting that the effects of gut microbiota may be associated with the NLRP3 inflammasome.

The NLRP3 inflammasome is a multiple protein complex consisting of nod-like receptor protein 3, adaptor protein ASC, and procaspase-1 precursor that was discovered and reported in 2002 (Martinon et al., 2002). It has been reported that the NLRP3 inflammasome can recognize the invasion of internal and external pathogens. Targeting NRLP3, as a therapeutic approach, results in activating caspase- 1 and promotes the maturation and secretion of pro-IL-1 $\beta$ and pro-IL-18, thus triggering the anti-pathogenic immune-inflammatory response of the body (Du et al., 2016). The NLRP3 inflammasome can be activated by several agents including bacteria, fungi, viral components, extracellular ATP, and silica crystals (Jo et al., 2015). Moderate activation of the NLRP3 inflammasome plays a role in several diseases including Alzheimer's disease, anxiety, and depression (He et al., 2019). Current evidence has promulgated the link between the NLRP3 inflammasome and gut microbes, indicating that changes in intestinal flora can impact the identification and response of inflammatory sensors and further regulate the activation of the NLRP3 inflammasome (Pérez-Figueroa et al., 2015).

Xiaoyaosan, a compound formula in traditional Chinese medicine (TCM), comprises eight crude herbs including Bupleurum chinense DC, Paeonia lactiflora Pall, Angelica sinensis (Oliv.) Diels, Atractylodes lancea (Thunb.) DC, Wolfiporia extensa (Peck) Ginns (syn. Poria cocos (Schwein.) F.A.Wolf), Glycyrrhiza glabra L., Mentha canadensis L., and Zingiber officinale Roscoe in a 5:5:5:5:5:4:1:5 ratio. This formulation was first described in a medical book, Taiping Huimin Heji Jufang, which was written during the Song Dynasty of China (960-1127 AD) (Li et al., 2017). Xiaoyaosan has been regarded as a remedy for liver-qi stagnation and spleen deficiency, while modern pharmacological studies have proven its efficacy in treating anxiety and depression (Zhang et al., 2012). Previous studies have suggested that Xiaoyaosan can improve depressive behavior in the CRS rat-model by regulation of the intestinal flora (Zhu et al., 2020). However, the specific mechanism by which Xiaoyaosan improves depressive behavior by modulating the intestinal flora and whether colon inflammation and NLRP3 inflammasome are related to the antidepressant mechanism of Xiaoyaosan need to be further studied. In this study, we aimed to investigate further whether Xiaoyaosan exerted antidepressant effects by modulating the gut microbiota and restraining the immoderate activation of the NLRP3 inflammasome in the colon.

\section{MATERIALS AND METHODS}

\section{Animals}

Sixty healthy 8-week-old male C57BL/6 SPF mice [SYXK (Yue) 2017-0174] weighing $20 \pm 2 \mathrm{~g}$ were purchased in advance, and they were subjected to a 7 -days adaptive feeding process before the start of the formal experiment. The experimental conditions were strictly implemented and included room temperature of $21 \pm 2^{\circ} \mathrm{C}$, relative humidity $30-40 \%$, and $12 \mathrm{~h}$ light $/ 12 \mathrm{~h}$ dark cycle. The animal experiments were reviewed by the Animal Ethics Committee (IACUC-20200810-04) and all experiments complied with the current animal-welfare guidelines.

\section{Behavioral Procedures}

Mice were randomly divided into four groups as follows: control, model, Xiaoyaosan, and probiotics. The model group was administered ampicillin ( $100 \mathrm{mg} / \mathrm{kg}$ and $0.1 \mathrm{ml} / 10 \mathrm{~g}$ body weight) in freshly diluted phosphate-buffered saline (PBS; pH 7.2), once daily for 14 consecutive days (Hyo-Min et al., 2018). The control group received an equivalent volume of saline and served as a negative control. The mice in the Xiaoyaosan group and the probiotic group received Xiaoyaosan $(0.658 \mathrm{~g} / \mathrm{kg} / \mathrm{d})$ and probiotics solution $(0.15 \mathrm{ml} /$ d) containing Bifidobacterium longum, Lactobacillus acidophilus, Bifidobacterium bifidum, Bifidobacterium breve, Bifidobacterium lactis, Lactobacillus brevis, Lactobacillus bulgaricus, Lactobacillus casei, Lactobacillus helveticus, Lactobacillus plantarum, Lactobacillus reuteri, Lactobacillus rhamnosus, Lactobacillus salivarius, Lactococcus lactis, Streptococcus thermophilus, and Bifidobacterium infantis (Figure 1). The weight of mice in each group was recorded one day before the start of the experiment on day 0 , and then on days 7 and 14 of the experiment, for comparison.

\section{Open Field Test}

The classic "open field" behavioral test method is mainly used to observe autonomous behavior, exploratory behavior, and stress of experimental animals in an unaccustomed environment. OFT was performed on days 0 and 14 in our study. Mice were placed in a behavioral operating room for $10 \mathrm{~min}$ for adaptation and then moved to the center zone. Camera recording was initiated and timed, and the behavior of the mice was observed for $5 \mathrm{~min}$. Immediately after each experiment, the boxes were cleaned with $75 \%$ alcohol. OFT was performed using the internationally recognized Behavior Analysis software (EthoVision software 


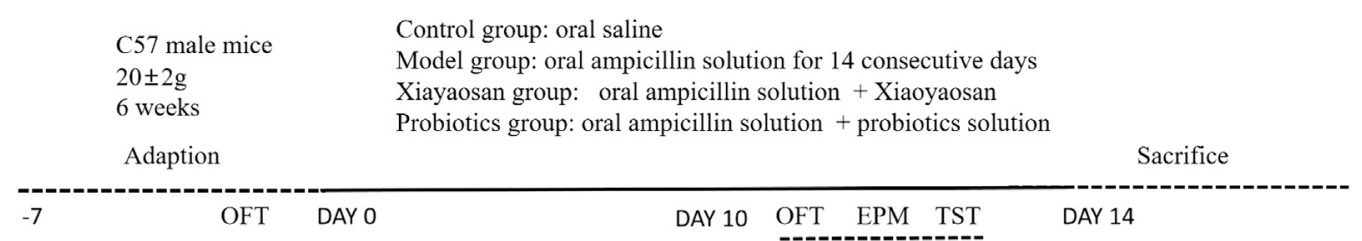

FIGURE 1 | Experimental flowchart. Mice were divided into four groups: the control group, model group, Xiaoyaosan treatment group, and probiotics treatment group. The model group received oral ampicillin solution for 14 consecutive days at a dose of $100 \mathrm{mg} / \mathrm{kg}$ and an intragastric volume of $0.1 \mathrm{ml} / 10 \mathrm{~g}$ body weight. The control group received oral saline in an equivalent volume and served as a negative control. Mice in the two treatment groups received Xiaoyaosan ( $0.658 \mathrm{~g} / \mathrm{kg} / \mathrm{d})$ and probiotics solution $(0.15 \mathrm{ml} / \mathrm{d})$, respectively.

analysis system, Noldus Information Technology) and included the analysis of the total movement distance of each group of mice (Choleris, 2001).

\section{Tail Suspension Test}

Mice were suspended on a horizontal rod $50 \mathrm{~cm}$ from the ground, with the tails fixed using adhesive tape. The entire experimental period lasted for $6 \mathrm{~min}$. The activity of each mouse for the last 4 min was recorded. Immobility time (seconds) was defined as the time required by the mice to give up struggling and remain completely motionless (Steru et al., 1985). The TST experiment data were analyzed using the Behavior Analysis software.

\section{Elevated Plus-Maze Test}

The EPM experiment was performed using a plus-maze device consisting of two open arms, two closed arms, and a central platform. The dimensions of the open and closed arms were both $30 \times 7 \mathrm{~cm}$, with the closed arms being covered by $20-\mathrm{cm}$ baffles on both sides. The mice were placed individually in the central area $(7 \times 7 \mathrm{~cm})$ at $60 \mathrm{~cm}$ above the ground, and their movements were recorded for $5 \mathrm{~min}$ (Rodgers and Johnson, 1995). Results of the EPM test were analyzed using the EthoVision software analysis system (Noldus Information Technology) and included the analysis of animal movement distance in open arms, duration time, and percentage of open arm duration time.

\section{Sample Collection and Preparation}

After the last behavioral test was completed, the mice were sacrificed using intraperitoneal injection of $3 \%$ sodium pentobarbital $(100 \mathrm{mg} / \mathrm{kg})$, and the feces were collected from the cecum in a sterile environment. The fecal samples were immediately stored at $20^{\circ} \mathrm{C}$ until DNA extraction. Colon tissues of mice were collected and stored for western blotting and qRT-PCR testing. The remaining colon tissues were stored in $4 \%$ paraformaldehyde solution at $4^{\circ} \mathrm{C}$ and subsequently used for tissue sectioning and immunohistochemistry.

\section{S Microbial Diversity Analysis}

The E.Z.N.A. Soil DNA Kit (Omega Bio-Tek, Norcross, GA, United States) was used to separate and extract the microbial DNA from the stool sample. The V3-V4 hypervariable region of the bacterial $16 \mathrm{~S}$ rRNA gene was amplified with the primers 338F ( $5^{\prime}$-ACTCCTACGGGAGGCAGCAG- $\left.3^{\prime}\right)$ and 806R (5'-GGACTACHVGGGTWTCTAAT- $\left.3^{\prime}\right)$. The PCR program was completed using a thermal cycling PCR system (GeneAmp 9700, ABI, United States) using the following program: denaturation at $95^{\circ} \mathrm{C}$ for $3 \mathrm{~min}$; at $95^{\circ} \mathrm{C}$ for 27 cycles of $30 \mathrm{~s}$; annealing at $55^{\circ} \mathrm{C}$ for $30 \mathrm{~s} ; 45 \mathrm{~s}$ for an extension at $72^{\circ} \mathrm{C}$; and final extension at $72^{\circ} \mathrm{C}$ for $10 \mathrm{~min}$. Trimmomatic was used to filter the quality of the original fastq files and merge the data using FLASH. The similarity of taxonomic units (OTUs) was classified using UPARSE (version 7.1, http://drive5.com/uparse/) according to 97\% criterion, and UCHIME was used to identify and remove chimeric sequences. Lastly, the RDP classifier algorithm (http:// rdp.cme.msu.edu/) program was executed on the Silva (SSU123) $16 \mathrm{~S}$ rRNA database using a $70 \%$ confidence threshold to analyze the classification of each 16S RNA gene sequence.

\section{Bioassays}

\section{H\&E Staining}

Samples of colonic tissue were fixed in $10 \%$ formalin, decalcified, dehydrated, made transparent, and then dipped and embedded in paraffin. Next, $5-\mu \mathrm{m}$-thick tissue samples were prepared using a microtome. Sections were dewaxed with xylene, passed through an aqueous ethanol series, stained using HE, and observed using microscopy.

\section{ELISA}

Serum LPS and IL- $1 \beta$ levels were determined using an ELISA detection kit (Cusabio Biotech., LTD., Wuhan, China). LPS levels in the colon homogenate and fecal supernatant were determined. LPS and IL-1 $\beta$ levels were determined by measuring the absorbance using a microplate reader and plotting a standard curve.

\section{Quantitative Real-Time PCR Analysis}

TRIzol reagent (Applied Biosystems, Waltham, MA, United States) was used to extract total RNA, and cDNA was synthesized using the RevertAid First Strand cDNA Synthesis Kit (Thermo Fisher Scientific, Waltham, MA, United States). The primers for NLRP3, ASC, and CASPASE-1 mRNA sequence used were as described in NCBI (shown in Table 1) and were synthesized by Sangon Biotech Co., Ltd (Shanghai, China). SYBR $^{\circledR}$ Green PCR Master Mix (Applied Biosystems) was used as the qRT-PCR reaction system with a volume of $25 \mu \mathrm{l}$ and detected using a Multicolor Real-time PCR detection system (BioRad, Hercules, CA, United States). The testing conditions were as follows: $95^{\circ} \mathrm{C}$ for $10 \mathrm{~min}$, followed by 40 cycles of $95^{\circ} \mathrm{C}$ for $30 \mathrm{~s}$ and 
TABLE 1 | Primer sequences used for qRT-PCR.

\begin{tabular}{|c|c|c|}
\hline Gene & Forward primer $(5,-3)$ & Reverse primer $(5,-3)$ \\
\hline NLRP3 & GAGCTGGACCTCAGTGACAATGC & ACCAATGCGAGATCCTGACAACAC \\
\hline ASC & GAAGTGGACGGAGTGCTGGATG & CTTGTCTTGGCTGGTGGTCTCTG \\
\hline CASPASE-1 & ACAACCACTCGTACACGTCTTGC & CCAGATCCTCCAGCAGCAACTTC \\
\hline GAPDH & TGAAGGGTGGAGCCAAAAG & AGTCTTCTGGGTGGCAGTGAT \\
\hline
\end{tabular}

$60^{\circ} \mathrm{C}$ for $1 \mathrm{~min}$. The $2^{-\Delta \Delta \mathrm{Ct}}$ method was used to calculate relative mRNA expression in each sample.

\section{Immunohistochemical Staining of Colon Tissues}

Immunohistochemical staining was used to characterize the paraffinembedded colon tissues. After washing, heating, dewaxing, dehydration, and antigen retrieval, the sections were treated with primary antibodies (NLRP3, Abcam, 1:150; ASC, Santa, 1:150; Caspase-1, Abcam, $1: 10$ ) at $37^{\circ} \mathrm{C}$ for $1 \mathrm{~h}$ and then at $4^{\circ} \mathrm{C}$ overnight. The slides were then incubated with the corresponding secondary antibodies (anti-mouse, Servicebio, United States; antirabbit, Servicebio, United States) at room temperature. The sections were developed using $\mathrm{DAB}$ (3,3-diaminobenzidine) reagent (Invitrogen, Carlsbad, CA, United States). After washing with PBS, the sections were re-stained with hematoxylin, dehydrated, and then photographed and recorded at $\times 400$ magnification (Leica, Leica Co., Tokyo, Japan). Mean Optical Density (MOD) was calculated using Image-Pro Plus software (version 6.0; Media Cybernetics, Bethesda, MD, United States).

\section{Western Blotting of Colon Tissue}

WB was used to determine the expression of NLRP3, ASC, and CASPASE-1 proteins in the colon tissue. A tissue protein extraction kit was used to extract total protein from the colon of each mouse and perform the BCA (Beyotime) protein assay. After electrophoresis and electroporation, the converted membrane was blocked with $5 \%$ skimmed milk powder for $1 \mathrm{~h}$, washed with TBST buffer, and incubated with the primary antibody overnight at $4^{\circ} \mathrm{C}$. The electrophoretic membrane was then incubated with a secondary antibody for $1 \mathrm{~h}$ and washed with TBST buffer; luminescence was measured using a chemiluminescence reagent (Millipore, Billerica, MA, United States). The protein-separation membrane was scanned and analyzed using an image analyzer (Bio-Rad, California, United States).

\section{Statistical Analysis}

Data are expressed as mean \pm SD and tested for normality and homogeneity of variance by using the SPSS25.0 software (Chicago, IL, United States). Data produced by repeated measurements were first analyzed via repeated analysis of variance (ANOVA), if the data were normally distributed and homogenous. If the data were not normally distributed or the variance was not uniform, the non-parametric test of $\mathrm{K}$ independent samples was used for item-by-item statistical analysis. A $p$-value $<0.05$ indicated significant differences for all the statistical tests. Drawing was performed using the GraphPad Prism 7.0 software (La Jolla, United States).

\section{RESULTS}

\section{Quality Control of Xiaoyaosan by UPLC-Q-TOF/MS}

Refer to the previous literature, the quality control of Xiaoyaosan was investigated by UPLC-Q-TOF/MS.(Xiaoyaosan is purchased from Jiuzhitang Group Co. Ltd., it has come from the same preparation and has the same batch number:20190724). The UPLC-Q-TOF/MS chromatogram of Xiaoyaosan is shown in Supplementary Figure S1A. As shown in Supplementary Figure S1B, seven compounds were distinguished: 1 . Paeoniflorin; 2. Kaempferol; 3. Quercetin; 4. Aloe emodin; 5. Luteolin; 6. Glyasperin C; 7. Acacetin.(Yuan et al., 2020).

\section{Effect of XYS on the Body Weight of Antibiotic-Induced Mice}

On day 0 and day 7 , there was no statistical difference in body weight across the four groups. However, on day 14, the weight of antibioticinduced mice group decreased significantly in comparison with that observed in the normal group $(p<0.01)$. Moreover, the body weight of the Xiaoyaosan group and probiotics group was higher than that of model group, but the difference was not significant (Figure 2A). The results suggested that the weight of the model group was significantly reduced after 14 days of antibiotic intervention while treatment with XYS or probiotics could resist antibiotic-induced weight loss.

\section{Effect of XYS on Antibiotic-Induced Depressive-Like Behavior in Mice}

To examine the effects of Xiaoyaosan on depressive-like behaviours, several behavioral tests including the tail suspension test (TST) and the open field test (OFT) were conducted.

For the TST test, antibiotic-induced mice showed increased immobility time in comparison with control mice $(p<0.01)$, recorded as the absence of escape-oriented behaviour in the TST (Figure 2B). For mice treated with Xiaoyaosan and probiotics, the immobility time in the experiment was remarkably reduced vs. the model group $(p<0.01)$.

For the OFT illustrated in Figure 2C, the heat map data shows the movement status of mice in open field experiments. As shown in Figure 2D, on day 0, the total distance travelled during $5 \mathrm{~min}$ was not significantly different when compared across the four groups. However, as shown in Figure 2E, on day 14, the antibioticinduced mice displayed a significant decrease in the total distance in comparison with the normal group $(p<0.001)$, suggesting that the model group was experiencing obvious depressive-like 

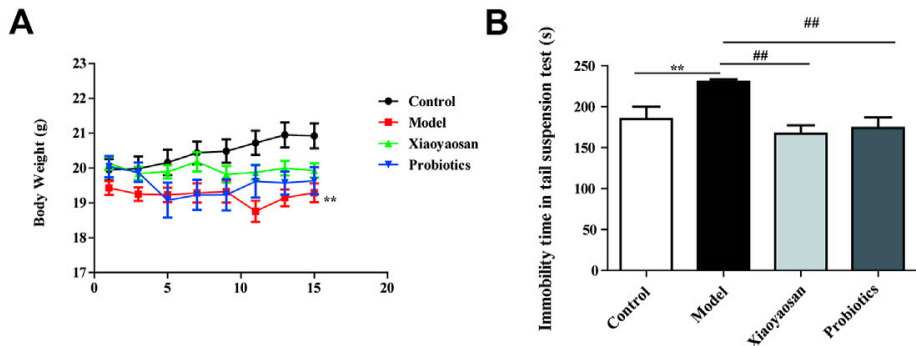

D
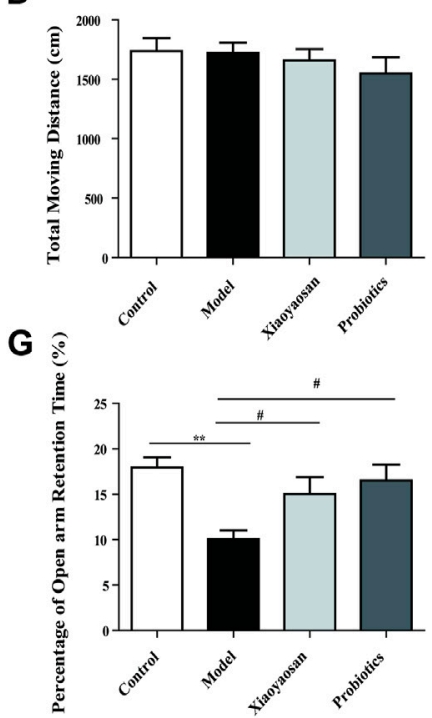

$\mathbf{E}$

H
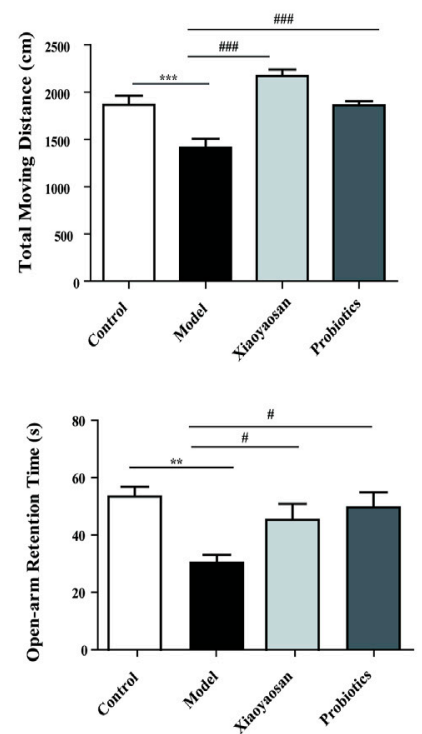

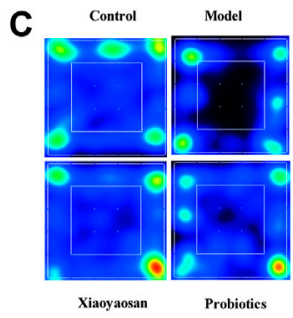

$\mathbf{F}$

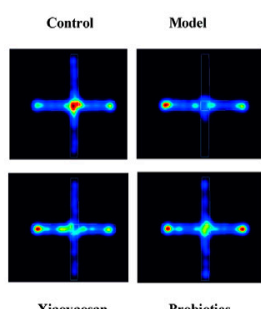

I

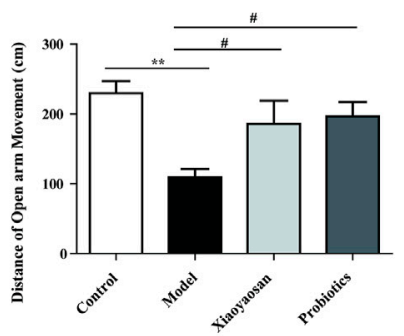

FIGURE 2 | Xiaoyaosan improves depressive-like behavior and anxiety-like behavior in antibiotic-induced mice. (A) Changes in body weight. (B) TST. (C) Heat map of OFT. (D) OFT on day 0. (E) OFT on day 14. (F) Heat map of FPM. (G, H, I) EPM. Data are expressed as the mean \pm SEM. ${ }^{* *} p<0.01,{ }^{* \star *} p<0.001$ vs. the control group; ${ }^{\#} p<0.05,{ }^{\# \#} p<0.01,{ }^{\# \# \#} p<0.001$ vs. the antibiotic-induced model group. $n=10$ per group.

behavior. However, this change was effectively improved by treatment with Xiaoyaosan $(p<0.001)$ and probiotics $(p<0.001)$.

\section{Effect of XYS on Antibiotic-Induced Anxiety-Like Behavior in Mice}

To examine the effects of Xiaoyaosan on anxiety-like behaviors, elevated plus-maze test (EPM) was conducted.

For the EPM illustrated in Figure 2F, the heat map data shows the movement status of mice in elevated plus-maze test. As illustrated in Figure 2G, in the EPM experiment, the percentage of time spent in open arms was significantly less in the model group than in the control group $(p<0.01)$. The percentage of time spent in open arms of Xiaoyaosan-treated animals was higher than in model, confirming the reduction in anxiety-like behaviors in rodents. In addition, the same results were obtained for the time spent in the open arms (Figure $\mathbf{2 H}$ ). In line with the above results, an obvious decrease in the distance travelled in the open arms (Figure 2I) was observed in the antibiotic-induced group vs. the control group. The results of the heat map (Figure 2B) also showed that the range of movement of antibiotic-induced mice was mainly concentrated in the closed arm, while the range of movement of Xiaoyaosan and probiotics was explored toward the open arm.

\section{Effect of XYS on Gut Microbiota of Antibiotic-Induced Mice}

The $16 \mathrm{~s}$ results showed that vs. the normal group, the gut microbiota of the model group mice were significantly different from the normal group at the species level (Figure 3A). Based on the results of a completely randomized multi-sample rank sum test, Lachnospiraceae, Bacteroidaceae, Akkermansia, Lachnoclostridium, Hungatella, Robinsoniella, Alloprevotella, Prevotellaceae, Parasutterella and other bacterial genera were significantly different. More important, Xiaoyaosan can significantly increase the abundance of Lachnospiraceae, and inhibit the growth of Bacteroidaceae (Figure 3B).

\section{Effects of XYS on the Level of LPS in Serum, Feces, Colon Homogenate and IL-1 $\beta$ Level of Serum in Antibiotic-Induced Mice}

To investigate the intention of Xiaoyaosan on flora-related signaling molecules, the level of LPS in serum, feces, colon 
A

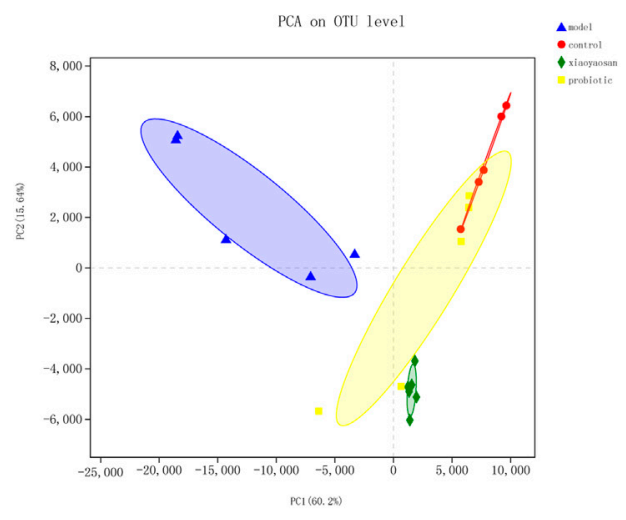

B

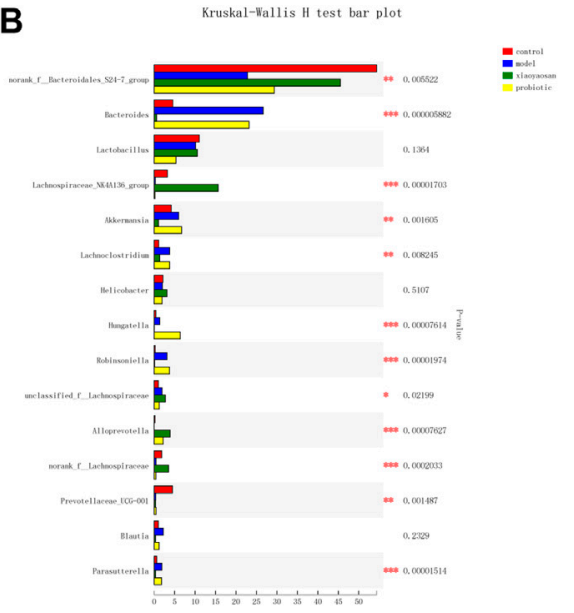

C

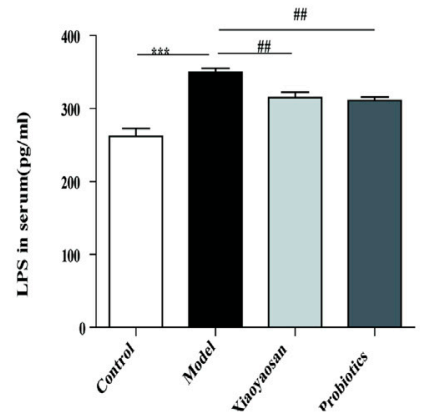

D

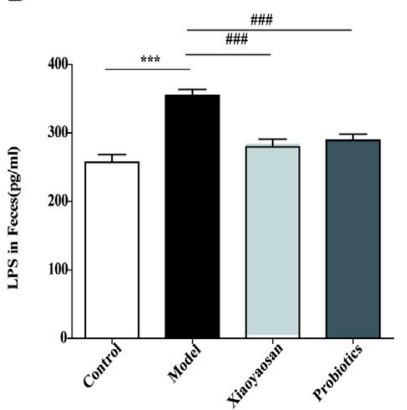

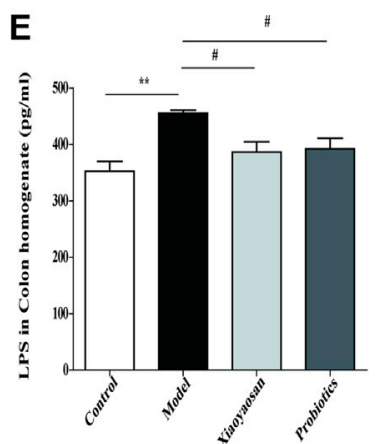

FIGURE 3 | Effect of XYS on gut microbiota and LPS Level of antibiotic-induced mice. (A) PCA principal component analysis. (B) Results of a completely randomized multi-sample rank sum test. (C) Effects of Xiaoyaosan on LPS Level of Serum. (D) Effects of Xiaoyaosan on LPS Level of feces. (E) Effects of Xiaoyaosan on LPS Level of colon homogenate.
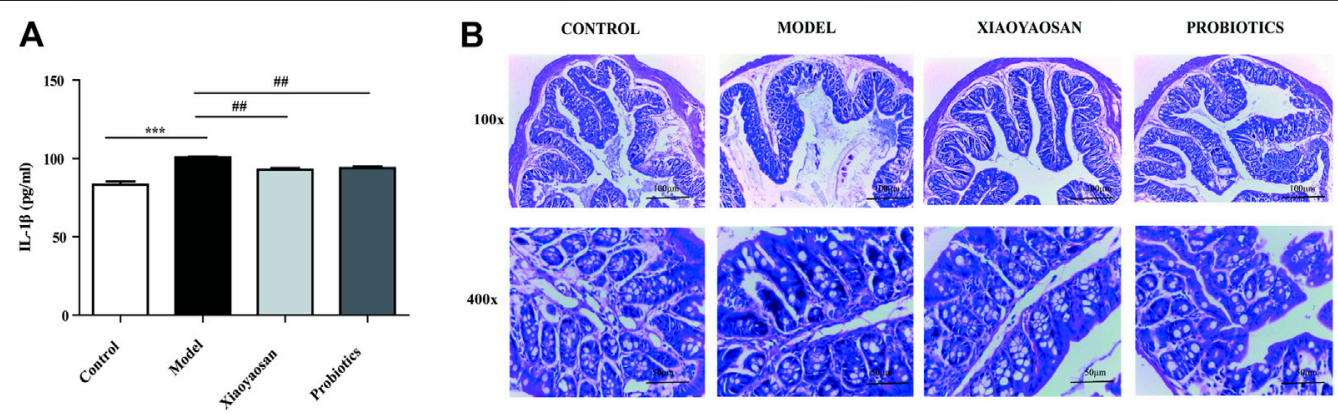

FIGURE 4 | Effects of Xiaoyaosan on IL-1 $\beta$ and Effect of Xiaoyaosan on Colon Pathology. (A) The IL-1 $\beta$ Level of Serum. (B) Effect of Xiaoyaosan on Colon Pathology. Hematoxylin and eosin staining of the colon (×100magnification, $\times 400$ magnification).

homogenate was determined. As shown in Figure 3C, the level of LPS in serum in the antibiotic-induced group were higher vs. the control group $(p<0.001)$, and Xiaoyaosan and probiotic Intervention significantly lessened the release of LPS ( $p<0.01$ and $p<0.01$, respectively) (Figures 3D,E). The same results were obtained for the level of LPS in feces and colon homogenate, indicating that after the intervention of antibiotics, there was an increase of LPS in the whole level of mice, which was significantly related to the intestinal flora, and this increase could be reversed by Xiaoyaosan and probiotics. Besides, we also determined the concentration of IL- $1 \beta$ in serum to observe the possible inflammatory response. As illustrated in Figure 4A, the serum IL-1 $\beta$ levels were remarkly higher in mice treated with antibiotic than in the control group $(p<0.001)$, and the Xiaoyaosan treatment reduced the levels $(p<0.01)$. 

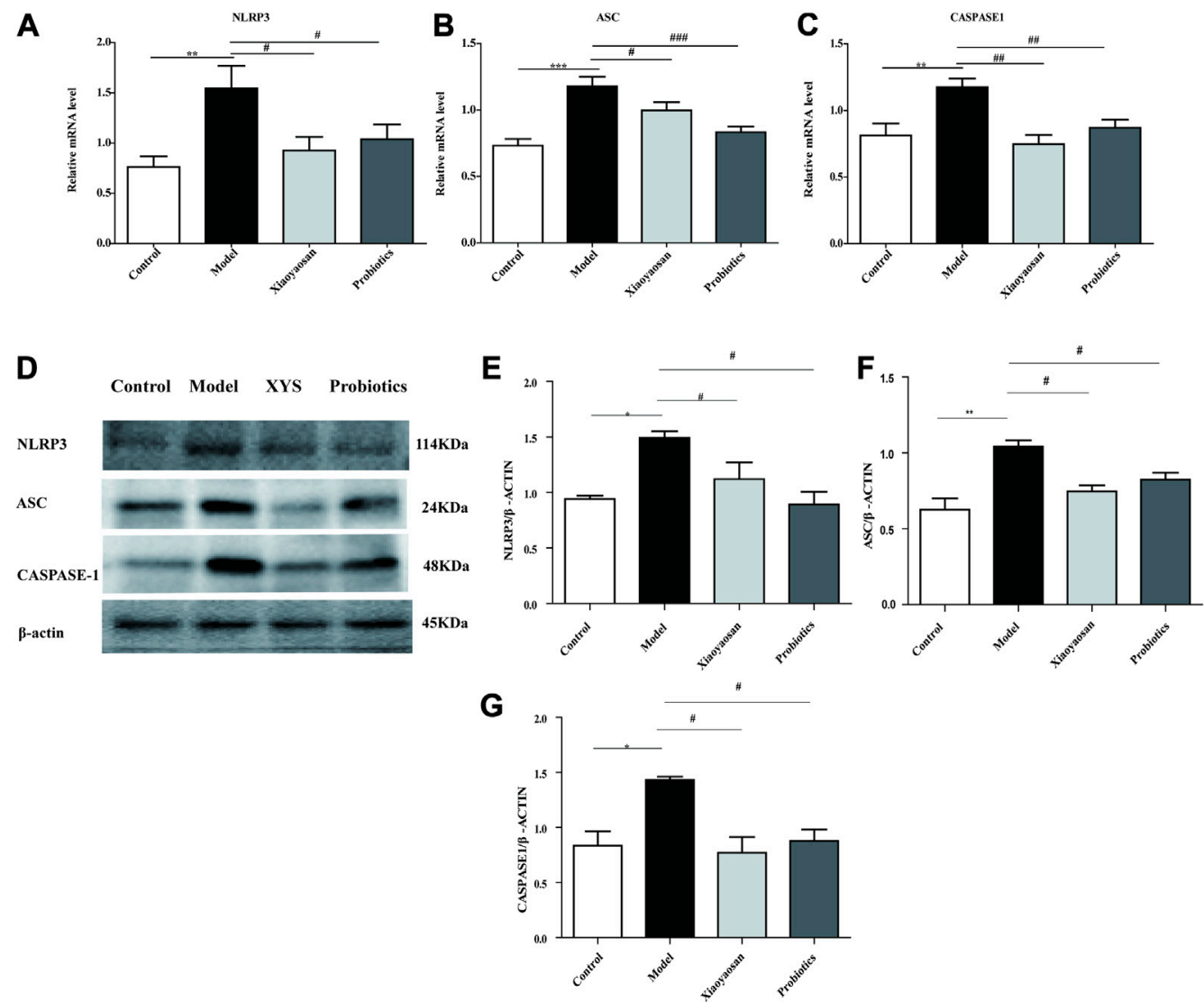

FIGURE 5 | Effects of Xiaoyaosan on the NLRP3-ASC-CASPASE-1 pathway in the colon. (A) NLRP3mRNA in colon ( $n=6)$. (B) ASCmRNA in colon ( $n=6)$. (C) CASPASE-1mRNA in colon ( $n=6)$. (D) Expression of NLRP3, ASC, CASPASE-1 protein in colon $(n=4)$. (E) NLRP3 protein in colon $(n=4)$. (F) ASC protein in colon $(n=$ 4). (G) CASPASE-1 protein in colon $(n=4)$. Data are expressed as the mean \pm SEM. ${ }^{*} p<0.05,{ }^{* \star} p<0.01$ vs. the control group; ${ }^{*} p<0.05$, ${ }^{\# \#} p<0.01$ vs. the antibioticinduced model group.

\section{Effect of XYS on Colon Pathology}

As illustrated in Figure $\mathbf{4 B}$, in the normal group, the colon mucosa was intact; epithelial cells were arranged neatly and there was no infiltration by inflammatory cells. In the model group, the colon mucosa was obviously, absent, the glands in the lamina propria were damaged or had disappeared, the number of goblet cells was reduced, and amount of inflammatory cells had infiltrated. In the Xiaoyaosan and probiotics group, the epithelial cell defects were not as obvious; furthermore, their basic morphology was similar to that of the normal group.

\section{Effects of XYS on the Expression of the NLRP3, Caspase-1 and ASC mRNAs in the Colon}

The relative expression levels of NLRP3, ASC and Caspase-1 genes were detected by PCR. There were significant differences in the mRNA expression of NLRP3, ASC and Caspase-1 units among groups. As illustrated in Figures 5A,B, vs. the model group, the expression of NLRP3 and ASC in the Xiaoyaosan group and the probiotics group decreased ( $p<0.05$ and $p<0.01$, respectively). As illustrated in Figure 5C, Compared with the model group, the relative expression of the Caspase- 1 gene in the Xiaoyaosan group and the probiotics group decreased significantly $(p<0.01)$.

\section{Effects of XYS on the Expression of the NLRP3, Caspase-1 and ASC Proteins in the Colon}

As illustrated in Figure 5D,E, the expression of NLRP3 was significantly increased in colon of antibiotic-induced mice, while was down-regulated by treatment with Xiaoyaosan or probiotic $(p<0.05)$. As illustrated in Figure 5F,G, vs. the model group, the expression of the ASC and Caspase- 1 in the Xiaoyaosan group and the probiotics group decreased sightly ( $p<0.05$ and $p<0.05$, respectively), together with the previous results, indicating that Xiaoyaosan inhibit the immoderate activation of the NLRP3 inflammasome in the colon.

\section{Effect of XYS on Immunohistochemistry of NLRP3, Caspase-1 and ASC in Colon}

In the model group, small brown granules representing NLRP3, Caspase-1 and ASC staining were significantly increased and strongly. For treatment of Xiaoyaosan and probiotics, the levels of 

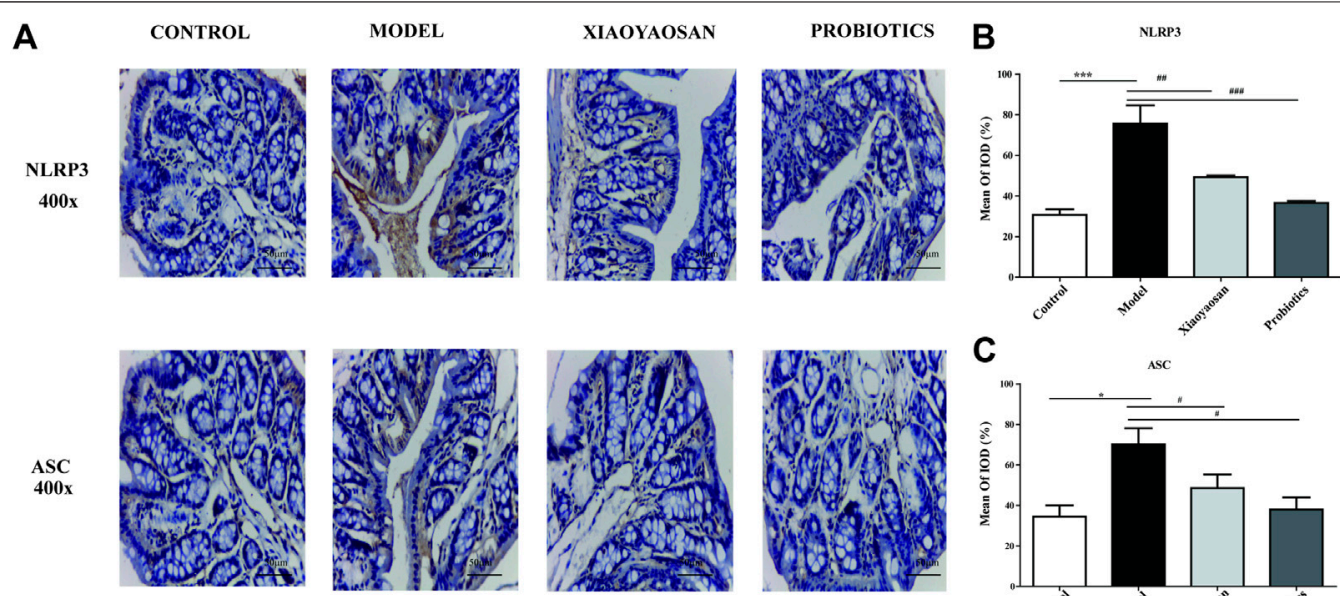

(1)
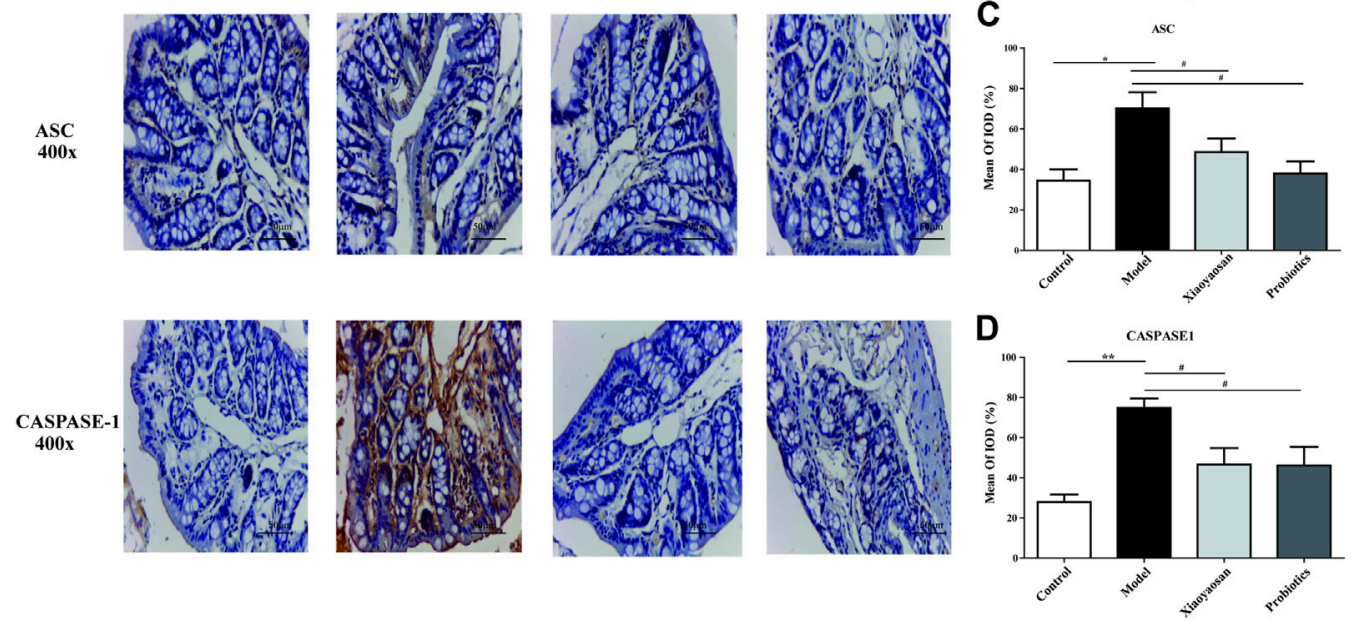

FIGURE 6 | Effects of Xiaoyaosan on the NLRP3-ASC-CASPASE-1 immunolabeling in the colon. (A) Immunohistochemical staining of NLRP3, ASC and CASPASE-1 protein in colon (scale bar $=50 \mathrm{~mm}, \times 400$ magnification). (B) Expression. of NLRP3 in colon. (C) Expression of ASC in colon. (D) Expression of CASPASE-1 in colon. $n=6$ per group. Data are expressed as the mean \pm SEM. ${ }^{*} p<0.05,{ }^{\star \star} p<0.01$ vs. the control group; ${ }^{\#} p<0.05$, ${ }^{\# \#} p<0.01$ vs. the antibiotic-induced model group.

NLRP3, Caspase-1 and ASC in each treatment group was significantly reduced (Figure 6A). IOD analysis showed that NLRP3 in the model group had increased significantly vs. the normal group $(p<0.001)$; Caspase- 1 and ASC in the model group had increased ( $p<0.05$ and $p<0.01$, respectively) (Figure 6B). Fxpression of NLRP3 in the Xiaoyaosan group and the probiotics group all showed significant reductions (Figures 6C,D); the levels of Caspase-1 and ASC in Xiaoyaosan group and probiotics group all showed slightly reduced levels $(p<0.05$ and $p<0.05$, respectively).

\section{DISCUSSION}

In this study, we investigated the regulatory effect of Xiaoyaosan in mouse models of depression and anxiety, using AIMD mice. The most important findings from this study are as follows: 1) Xiaoyaosan improved depressive and anxious behavior in AIMD mice. 2) Treatment with Xiaoyaosan altered the gut microbiota while reducing LPS levels in the intestinal and systemic circulation. 3) Xiaoyaosan may exert antidepressant-like and anxiolytic effects by restraining the moderate activation of the NLRP3 inflammasome in the colon.

The gut microbiota plays a significant role in the development of mental illnesses including depression and anxiety. The twoway regulation of the brain-gut axis is a vital mechanism by which gut microbiota affect the central nervous system (CNS). Through interaction between the enteric nervous system, vagus nerve, HPA axis, microbial metabolites, host signal molecules, and immune factors, gut microbiota can affect the development and regulation of CNS, achieve bottom-up regulation of CNS, and participate in the pathological processes of various mental diseases, including depression and anxiety (Rieder et al., 2017). Preclinical studies have shown that the long-term use of antibiotics can cause behavioral disorders in patients, with symptoms similar to those of depression and anxiety. Moreover, the AIMD mouse model is widely used to assess the role of the intestinal flora and efficacy of antidepressants or anxiolytics in rodents (Merzoug et al., 2002). Clinical studies have confirmed that antibiotic intervention and disturbance in intestinal flora can increase the risk of depression and anxiety (Lurie et al., 2015). The AIMD mouse model represents disorders of the intestinal flora, accompanied by behavioral changes including depression, anxiety, social disorders, and aggressive consciousness (Hao et al., 2020), and indicates a possible close relationship between intestinal flora in humans and their behavior (Leulier et al., 2017). In this study, we used an ampicillin-induced model to study anxiety and depressive behavior, and used $16 \mathrm{~S}$ technology to determine changes in the gut microbiota. Our findings were identical to those reported in previous studies (Liang et al., 2017). We have also reproduced the antidepressant and anti-anxiety effects of Xiaoyaosan by using the chronicre straint strees and chronic unpredictable mild stress models (Guo et al., 2017), suggesting 
that treatment with Xiaoyaosan alters the gut microbiota, and confirming that the gut microbiota is an important target for Xiaoyaosan.

In this study, we found that Xiaoyaosan could alter microbial abundance after antibiotic intervention, reduce LPS levels, and affect intestinal inflammation, thereby providing new evidence for gut flora as a therapeutic target in the management of mental disorders. The intestinal flora plays a vital role in host metabolism and the maintenance of homeostasis of the gastrointestinal tract (Round and Mazmanian, 2009). When there is an imbalance in the gut microbiota, certain microbes can mediate intestinal metabolic imbalance and intestinal inflammation, induce metabolic diseases, and cause mental illness. Bacteroidaceae is a gram-negative bacterium comprising the intestinal microflora. Many clinical studies have reported a high abundance of Bacteroidaceae in the stools of patients with depression, anxiety, hyperlipidemia, and liver cirrhosis, suggesting that the presence of Bacteroidaceae may be associated with the development of mental and metabolic diseases (Xie et al., 2016). Bacteroidaceae are also the main producer of LPS, which is associated with the activation of inflammation and participation in a series of inflammatory diseases including colitis and hepatitis (Whitfield and Trent, 2014). In this study, we found that Xiaoyaosan could change the abundance of specific genera in the intestinal flora, which was manifested by a significant increase in the abundance of Lachnospiraceae and inhibition of the growth of Bacteroidaceae. When combined with the metagenomics and gut microbiota data of a previous experiment (Hui-Zheng et al.), we found that Xiaoyaosan could significantly reduce the level of LPS in mouse stools, colon homogenate, and serum. These findings demonstrated that abnormal LPS levels in the intestinal and peripheral circulation were corrected after the restoration of the intestinal flora. This study also revealed a relationship between LPS and colon inflammation. AIMD mice exhibited significant colonic inflammation and increased LPS levels, which were markedly reduced after treatment with Xiaoyaosan, resulting in an improved inflammatory status of the colon. This result corroborates previous findings and highlights the role of LPS in inflammation. To summarize, our findings suggested that the reduction of LPS and the improvement of colon inflammation mediate the antidepressant and anxiolytic effects of Xiaoyaosan.

We found that Xiaoyaosan could influence antibiotic-induced depressive behavior by inhibiting NLRP3 inflammasome activation in the colon. The NLRP3 inflammasome can be activated by LPS to induce caspase- 1 cleavage, leading to the maturation of the proinflammatory cytokines IL-1 $\beta$ and IL-18 (Ye et al., 2016). Several studies have demonstrated that the NLRP3 inflammasome is involved in the pathogenesis of depression and anxiety (Alcocer-Gómez and Cordero, 2014). Activation of NLRP3 and IL- $1 \beta$ is observed in patients with depression and anxiety (AlcocerGómez et al., 2016). Thus, targeting the NLRP3 inflammasome may be a new approach for treating depression (Du et al., 2016). In this study, we used AIMD mice and found that ampicillin activated the NLRP3 inflammasome in the colon and released a large amount of IL-1 $\beta$ in the colon and serum. Xiaoyaosan treatment abolished the antibiotic-induced activation of the NLRP3 inflammasome and improved depressive and anxious behavior, as evidenced by a striking increase in the total distance in the OFT and a decrease in the duration of immobility. This evidence indicates that inhibiting NLRP3 inflammasome activation may result in antidepressant and anxiolytic effects similar to those observed in Xiaoyaosan-treated mice.

The two-way regulatory effect of Xiaoyaosan on depression and anxiety has been previously reported. For example, Xiaoyaosan can exert anxiolytic effects through the CRF1 receptor (Jiang et al., 2016) and the JNK signaling pathway (Zhao et al., 2017) and can improve depressive behavior by targeting the Nesfatin-1 receptor (Ma et al., 2019). Our current research confirmed the two-way regulation of anxiety

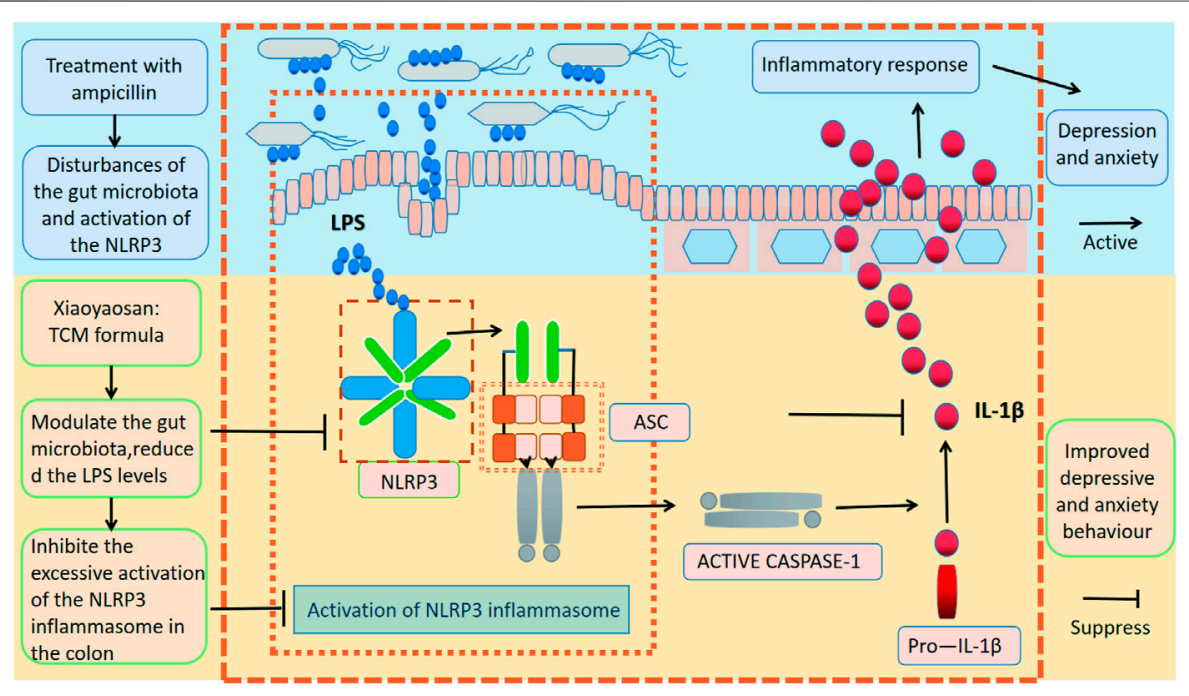

FIGURE 7 | Xiaoyaosan suppresses anxiolytic-like behaviors and improve depressive behavior by modulating the gut microbiota and inhibiting the excessive activation of the NLRP3 inflammasome in the colon. 
and depression by Xiaoyaosan could be attributed to the alteration in the abundance of intestinal flora and reduction in endotoxin-induced inflammation.

Our study has some limitations. First, Xiaoyaosan contains multiple Chinese herbal medicines, and its ingredients are complex; we have only demonstrated its antidepressant and anxiolytic effects in combination (Gao et al., 2018). The activity and synergy between components are not yet clear, and further studies are needed to elucidate the component or components responsible for the effects we have observed. Second, the results of our study suggest that the levels of LPS and inflammatory factors in the systemic circulation increase but whether this directly affects the brain through the blood-brain barrier has not been addressed. More importantly, the direct evidence that Xiaoyaosan improves depression-like behaviors through intestinal flora needs to be further studied. Therefore, in future work, we will use fecal microbiota transplantation (FMT) and germ-free mice (GF) to further study the antidepressant and anti-anxiety effects of Xiaoyaosan based on intestinal microbes. We plan to focus on this aspect in our future research.

\section{CONCLUSION}

We have demonstrated a potential role of the gut microbiota in the antidepressant and anxiolytic effects of Xiaoyaosan in the AIMD mouse model (Figure 7), thereby enriching our understanding of the pharmacology of Xiaoyaosan. This innovative research has revealed the antidepressant and antianxiety effects of Xiaoyaosan through its effect on endotoxin metabolism disorder caused by intestinal flora and the excessive activation of the NLRP3 inflammasome. Our findings suggest that Xiaoyaosan may improve anxiolytic and depressive behavior by modulating the gut microbiota and inhibiting the immoderate activation of the NLRP3 inflammasome in the colon, offering further evidence for the clinical application of Xiaoyaosan.

\section{DATA AVAILABILITY STATEMENT}

The datasets presented in this study can be found in online repositories. The names of the repository/repositories and

\section{REFERENCES}

Alcocer-Gómez, E., and Cordero, M. D. (2014). NLRP3 inflammasome: a new target in major depressive disorder. Cns Neurosci. Ther. 20 (3), 294-295. doi:10. $1111 / \mathrm{cns} .12230$

Alcocer-Gómez, E., Ulecia-Morón, C., Marín-Aguilar, F., Rybkina, T., Casas-Barquero, N., Ruiz-Cabello, J., et al. (2016). Stressinduced depressive behaviors require a functional NLRP3 inflammasome. Mol. Neurobiol. 53 (7), 4874-4882. doi:10.1007/ s12035-015-9408-7

Baxter, A. J., Patton, G., Scott, K. M., Degenhardt, L., and Whiteford, H. A. (2014). Global epidemiology of mental disorders: what are we missing? PLos One 8 (6), e65514. doi:10.1371/journal.pone.0065514 accession number(s) can be found below: NCBI SRA BioProject, accession no: PRJNA716118.

\section{ETHICS STATEMENT}

The animal study was reviewed and approved by Jinan University.

\section{AUTHOR CONTRIBUTIONS}

$\mathrm{WH}$ and JC main contributed research conception and design; WH, JW, NY, LG, HZ, HG, XD, JH, LD, XL, and QM collected and processed data, $\mathrm{WH}$ wrote sections of the manuscript. All authors contributed to manuscript revision, read and approved the final submitted version. The corresponding author JC takes primary responsibility for communication with the journal and editorial office during the submission process, throughout peer review and during publication.

\section{FUNDING}

The work was supported by the Project of National Natural Science Foundation of China (grant numbers 81630104, 81973748, 81803998, and 82074300) Huang Zhendong Research Fund for Traditional Chinese Medicine of Jinan University.

\section{ACKNOWLEDGMENTS}

The authors gratefully acknowledged the support of K. C. Wong Education Foundation.

\section{SUPPLEMENTARY MATERIAL}

The Supplementary Material for this article can be found online at: https://www.frontiersin.org/articles/10.3389/fphar.2021.619103/ full\#supplementary-material.

Chen, Z., Li, J., Gui, S., Zhou, C., Chen, J., Yang, C., et al. (2018). Comparative metaproteomics analysis shows altered fecal microbiota signatures in patients with major depressive disorder. Neuroreport 29 (5), 1. doi:10.1097/wnr.0000000000000985

Choleris, E. (2001). A detailed ethological analysis of the mouse open field test: effects of diazepam, chlordiazepoxide and an extremely low frequency pulsed magnetic field. Neurosci. Biobehavioral Rev. 25 (3), 235-260. doi:10.1016/ s0149-7634(01)00011-2

Du, R.-H., Tan, J., Sun, X.-Y., Lu, M., Ding, J.-H., and Hu, G. (2016). Fluoxetine inhibits NLRP3 inflammasome activation: implication in depression. Ijnppy 19 (9), pyw037. doi:10.1093/ijnp/pyw037

Guo, X., Qiu, W., Liu, Y., Zhang, Y., Zhao, H., and Chen, J. (2017). Effects of refined xiaoyaosan on depressive-like behaviors in rats with chronic unpredictable mild stress through neurosteroids, their Synthesis and metabolic enzymes. Molecules 22 (8), 1386. doi: $10.3390 /$ molecules 22081386 
Hao, W.-Z., Li, X.-J., Zhang, P.-W., and Chen, J.-X. (2020). A review of antibiotics, depression, and the gut microbiome. Psychiatry Res., 284:112691. doi:10.1016/j. psychres.2019.112691

He, W., Long, T., Pan, Q., Zhang, S., Zhang, Y., Zhang, D., et al. (2019), Microglial NLRP3 inflammasome activation mediates IL-1 $\beta$ release and contributes to central sensitization in a recurrent nitroglycerin-induced migraine model. J. Neuroinflamm. 16 (1), 78. doi:10.1186/s12974-019-1459-7

Hyo-Min, J., Lee, H.-J., Jang, S.-E., Han, M. J., and Kim, D. H., (2018), Evidence for interplay among antibacterial-induced gut microbiota disturbance, neuroinflammation, and anxiety in mice. Mucosal Immunol. 11(5):1386-1397. doi:10.1038/s41385-018-0042-3

Jiang, Y.-M., Li, X.-J., Meng, Z.-Z., Liu, Y.-Y., Zhao, H.-B., Li, N., et al. (2016). Effects of xiaoyaosan on stress-induced anxiety-like behavior in rats: involvement of CRF1 receptor. Evidence-Based Complement. Altern. Med. 2016, 1-9. doi:10.1155/2016/1238426

Jo, E.-K., Kim, J. K., Shin, D.-M., and Sasakawa, C. (2015). Molecular mechanisms regulating NLRP3 inflammasome activation. Cell Mol Immunol 13 (2), 148-159. doi:10.1038/cmi.2015.95

Kessler, R. C., and Bromet, E. J. (2013). The epidemiology of depression across cultures. Annu. Rev. Public Health 34 (1), 119-138. doi:10.1146/annurevpublhealth-031912-114409

Leulier, F. O., MacNeil, L. T., Lee, W.-J., Rawls, J. F., Cani, P. D., Schwarzer, M., et al. (2017). Integrative physiology: at the crossroads of nutrition, microbiota, animal physiology, and human health. Cell Metab. 25 (3), 522-534. doi:10.1016/ j.cmet.2017.02.001

Li, X. J., Ma, Q. Y., Jiang, Y. M., Bai, X. H., Yan, Z. Y., Liu, Q., et al. (2017). Xiaoyaosan exerts anxiolytic-like effects by down-regulating the TNF- $\alpha$ /JAK2STAT3 pathway in the rat hippocampus. Sci. Rep. 7 (1), 353. doi:10.1038/ s41598-017-00496-y

Liang, L., Zhou, H., Zhang, S., Yuan, J., and Wu, H. (2017). Effects of gut microbiota disturbance induced in early life on the expression of extrasynaptic GABA-A receptor $\alpha 5$ and $\delta$ subunits in the hippocampus of adult rat. Brain Res. Bull. 135, 113-119. doi:10.1016/j.brainresbull.2017.09.014

Gao, L., Huang, P., Dong, Z., Gao, T., Huang, S., Zhou, C., et al. (2018). Modified xiaoyaosan (MXYS) exerts anti-depressive effects by rectifying the brain blood oxygen level-dependent fMRI signals and improving hippocampal neurogenesis in mice. Front. Pharmacol. 9, 1098. doi:10.3389/fphar.2018.01098

Lurie, I., Yang, Y.-X., Haynes, K., Mamtani, R., and Boursi, B. (2015). Antibiotic exposure and the risk for depression, anxiety, or psychosis. J. Clin. Psychiatry 76 (11), 1522. doi:10.4088/jcp.15m09961

Ma, Q., Li, X., Yan, Z., Jiao, H., Wang, T., Hou, Y., et al. (2019). Xiaoyaosan ameliorates chronic immobilization stress-induced depression-like behaviors and anorexia in rats: the role of the nesfatin-1-oxytocin-proopiomelanocortin neural pathway in the hypothalamus. Front. Psychiatry 12 (10) doi:10.3389/ fpsyt.2019.00910

Martinon, F., Burns, K., and Tschopp, J. (2002). The inflammasome. Mol. Cell 10 (2), 417-426. doi:10.1016/s1097-2765(02)00599-3

Merzoug, S., Toumi, M. L., and Tahraoui, A., (2002). Quercetin mitigates Adriamycin-induced anxiety- and depression-like behaviors, immune dysfunction, and brain oxidative stress in rats. Naunyn-Schmiedeberg's Archives Pharmacol. 387 (10), 921-933. doi:10.1007/s00210-014-1008-y

Pérez-Figueroa, E., Torres, J., Sánchez-Zauco, N., Contreras-Ramos, A., AlvarezArellano, L., and Maldonado-Bernal, C. (2015). Activation of NLRP3 inflammasome in human neutrophils byHelicobacter pyloriinfection. Innate Immun. 22 (2), 103. doi:10.1177/1753425915619475

Rieder, R., Wisniewski, P. J., Alderman, B. L., and Campbell, S. C. (2017). Microbes and mental health: a review. Brain Behav. Immun. 66, 9-17. doi:10.1016/j.bbi. 2017.01.016
Rodgers, R. J., and Johnson, N. J. T. (1995). Factor analysis of spatiotemporal and ethological measures in the murine elevated plus-maze test of anxiety. Pharmacol. Biochem. Behav. 52 (2), 297-303. doi:10.1016/0091-3057(95) 00138-m

Round, J. L., and Mazmanian, S. K. (2009). The gut microbiota shapes intestinal immune responses during health and disease. Nat. Rev. Immunol. 9 (8), 313-323. doi:10.1038/nri2515

Sender, R., Fuchs, S., and Milo, R. (2016). Are we really vastly outnumbered? Revisiting the ratio of bacterial to host cells in humans. Cell 164 (3), 337-340. doi:10.1016/j.cell.2016.01.013

Sherwin, E., Dinan, T. G., and Cryan, J. F. (2018). Recent developments in understanding the role of the gut microbiota in brain health and disease. Ann. N. Y Acad. Sci. 1420 (1). doi:10.1111/nyas.13416

Slykerman, R. F., Hood, F., Wickens, K., Thompson, J. M. D., Barthow, C., Murphy, R., et al. (2017). Effect of Lactobacillus rhamnosus HN001 in pregnancy on postpartum symptoms of depression and anxiety: a randomised double-blind placebo-controlled trial. Ebiomedicine 24:159-165. doi:10.1016/j.ebiom.2017. 09.013

Steru, L., Chermat, R., Thierry, B., and Simon, P. (1985). The tail suspension test: a new method for screening antidepressants in mice. Psychopharmacology 85 (3), 367. doi:10.1007/bf00428203

Whitfield, C., and Trent, M. S. (2014). Biosynthesis and export of bacterial lipopolysaccharides. Annu. Rev. Biochem. 83 (1), 99-128. doi:10.1146/ annurev-biochem-060713-035600

Xie, G., Wang, X., Liu, P., Wei, R., Chen, W., Rajani, C., et al. (2016). Distinctly altered gut microbiota in the progression of liver disease. Oncotarget 7 (15), 19355. doi:10.18632/oncotarget.8466

Ye, C., Li, S., Yao, W., Xu, L., Qiu, Y., Liu, Y., et al. (2016). The anti-inflammatory effects of baicalin through suppression of NLRP3 inflammasome pathway in LPS-challenged piglet mononuclear phagocytes. Innate Immun. 22 (3), 196-204. doi:10.1177/1753425916631032

Yuan, N, Gong, L., Tang, K., He, L., Hao, W., Li, , X., et al. (2020). An integrated pharmacology-based analysis for antidepressant mechanism of Chinese herbal formula xiao-yao-san. Front. Pharmacol., 11

Zhang, Y., Han, H., Liu, Z., Wang, J., He, Q., and Liu, J. (2012). Chinese herbal formula xiao yao san for treatment of depression: a systematic review of randomized controlled trials. Evid Based. Complement. Altern. Med. 2012 (1741-427X), 931636. doi:10.1155/2012/931636

Zhao, H.-B., Jiang, Y.-M., Li, X.-J., Liu, Y.-Y., Bai, X.-H., Li, N., et al. (2017). Xiao yao san improves the anxiety-like behaviors of rats induced by chronic immobilization stress: the involvement of the JNK signaling pathway in the Hippocampus. Biol. Pharm. Bull. 40 (2), 187-194. doi:10.1248/bpb.b16-00694

Zhu, H.-Z., Liang, Y.-D., Ma, Q.-Y., Hao, W.-Z., Li, X.-J., Wu, M. S., et al. (2020). Xiaoyaosan improves depressive-like behavior in rats with chronic immobilization stress through modulation of the gut microbiota. Biomed. Pharmacother. 112:108621. doi:10.1016/j.biopha.2019.108621

Conflict of Interest: The authors declare that the research was conducted in the absence of any commercial or financial relationships that could be construed as a potential conflict of interest.

Copyright (C) 2021 Hao, Wu, Yuan, Gong, Huang, Ma, Zhu, Gan, Da, Deng, Li and Chen. This is an open-access article distributed under the terms of the Creative Commons Attribution License (CC BY). The use, distribution or reproduction in other forums is permitted, provided the original author(s) and the copyright owner(s) are credited and that the original publication in this journal is cited, in accordance with accepted academic practice. No use, distribution or reproduction is permitted which does not comply with these terms. 